\title{
Compressive and Tensile Axial Strain reduced Critical Currents in Bi-2212 Conductors
}

\author{
Bennie ten Haken and Herman H. J. ten Kate. \\ Applied Superconductivity Centre, University of Twente, P.O. Box 217, 7500 AE Enschede, The Netherlands \\ J. Tenbrink \\ Vacuum Schmeltze GmbH, Grunerweg 37, D-6450 Hanau 1, Germany
}

\begin{abstract}
Mono and multifilamentary wires of BSCCO2212 in Ag matrix are investigated in an axial strain experiment. The superconducting samples are soldered to a substrate that is bend in order to achieve a compressive or tensile axial strain. The $I_{c}$-strain dependence is measured in magnetic fields up to $16 \mathrm{~T}$ at $4.2 \mathrm{~K}$ and the strain is varied from $-2 \%$ to $+1.2 \%$. In these $\mathrm{Bj}-\mathbf{2 1 2 2}$ samples any strain-induced $I_{c}$ reduction is irreversible. Moreover a significant rise in $I_{c}$ was never observed after changing the strain. Special attention is paid to the tensile axial strain regime (0 to $0.4 \%$ ). A small but significant reduction in $I_{c}$ is found in this case. The strain behaviour of these wires indicates that the $I_{c}$ reduction is due to fractures in the superconducting filaments.
\end{abstract}

\section{INTRODUCTION}

The abbreviation " $\mathrm{Bi}-2212$ " denotes the superconducting material $\mathrm{Bi}_{2} \mathrm{Sr}_{2} \mathrm{CaCu}_{2} \mathrm{O}_{8+\delta}$. Within the family of copperoxide superconductors, the $\mathrm{Bi}-2212$ material is at present a promising member to realise high-field applications at low temperatures in the near future. The superconducting wires and tapes are produced with the "powder in tube" (PIT) method [1]. The influence of mechanical strain on the superconducting properties is investigated by means of a Ushaped bending spring. In this setup the wire is soldered with indium onto the brass substrate, that is bend in order to deform the wire. An advantage of this apparatus is that both compressive and tensile axial strains can be induced in the same sample [2].

\section{SAMPLE MATERIAL}

The various conductor batches are identified by a single character with and an integer for the number of filaments (e.g. A-1, C-19, G-55). A survey is presented in table I. The samples are untwisted pieces of conductor with a length of about $50 \mathrm{~mm}$ and a diameter of $1 \mathrm{~mm}$. The wires have either $\mathrm{Ag}$ matrix or a $\mathrm{AgNiMg}$ matrix with a higher yield strength in order to increase the mechanical strength. The microstructure shows no major texturing in the $\mathrm{Bi}-2212$ phase after reaction and a low filling factor of $70 \%$ in the granular structure. The critical current density in the non-silver area of the Bi-2212 wires is 500 to $1000 \mathrm{~A} / \mathrm{mm}^{2}$ at $4.2 \mathrm{~K}$ and a factor of 100 lower at $77 \mathrm{~K}$, with $B=0$ in both cases [2].

Manuscript received October 17, 1994
TABLE I

A SURVEY OF THE BI-2212 SAMPLES INVESTIGATED.

\begin{tabular}{|c|c|c|c|c|c|}
\hline Sample & Matrix & Non-Ag & Sample & Matrix & Non-Ag \\
\hline A-1 & $\mathrm{Ag}$ & $0.23 \mathrm{~mm}^{2}$ & C-19 & AgNiMg & $0.25 \mathrm{~mm}^{2}$ \\
\hline A-19 & $\mathrm{Ag}$ & $0.29 \mathrm{~mm}^{2}$ & C-37 & $\mathrm{AgNiMg}$ & $0.19 \mathrm{~mm}^{2}$ \\
\hline B-1 & $\mathrm{Ag}$ & $0.23 \mathrm{~mm}^{2}$ & F-19 & AgNiMg & $0.27 \mathrm{~mm}^{2}$ \\
\hline B-19 & $\mathrm{Ag}$ & $0.20 \mathrm{~mm}^{2}$ & G-37 & $\mathrm{AgNiMg}$ & $0.19 \mathrm{~mm}^{2}$ \\
\hline B-37 & Ag & $0.19 \mathrm{~mm}^{2}$ & H-55 & Ag & $0.17 \mathrm{~mm}^{2}$ \\
\hline
\end{tabular}

\section{TENSILE STRAIN}

A comparison of the axial-strain measurements on the various wires is presented in figure 1 . The critical current as a function of the axial strain $\left(I_{c}\left(\varepsilon_{a}\right)\right)$ has a comparable shape as found elsewhere in similar conductors [3]. First there is a strain regime where the critical current reduction is small $(<2 \%)$. For higher strain values there occurs a strong and certainly irreversible $I_{c}$ reduction.

Sometimes a so-called irreversible strain limit $\left(\varepsilon_{i r r}\right)$ is defined based on the observed $I_{c}\left(\varepsilon_{a}\right)$ relation. For these samples this results for instance in an $\varepsilon_{i r r}$ of $0.40 \%$. The definition of this strain limit depends highly on the accuracy of the $I_{c}$ determination. In order to reduce the influence of the experimental accuracy, and to avoid discussions about what is "constant" and "irreversible" an alternative parameter for the irreversible strain is defined here as: $\varepsilon_{2 \%}$, being the strain where a reduction of $2 \%$ in $I_{c}$ is observed.

In order to achieve a reasonable accuracy in the $I_{c}$ determination a criterion of $10^{-3} \mathrm{~V} / \mathrm{m}$ is applied which leads to a reproducibility of $\pm 0.2 \%$. This accuracy is mainly determined by the voltage noise $( \pm 50 \mathrm{nV})$ in combination with a limited sample length $(\approx 10 \mathrm{~mm})$ and the low $n$-value of these wires $(\approx 4)$. The more common $I_{c}$ criterion of $10^{-4} \mathrm{~V} / \mathrm{m}$ leads to a reproducibility of $\pm 2 \%$. In that case the values of $\varepsilon_{2 \%}$ and $\varepsilon_{i r r}$ will coincide in this experimental setup. Within the experimental accuracy the shape of the $I_{c}\left(\varepsilon_{a}\right)$ curve is similar for both criterias [4].

\section{A. High Tensile Strain}

Two different types of matrix material are involved. The wires with the AgNiMg matrix (C, F \& G) behave mechanically different because of the higher strength of the alloyed 
silver. On the bending spring the axial strain is measured and therefore a different mechanical strength cannot be detected. The following classification can be made:

1: The C, F \& G samples with the AgNiMg matrix show a relatively small decrease in $I_{c}$ beyond the $\varepsilon_{2 \%}$, with a relative slope of $\approx 120$ (percent $I_{c}$ reduction per percent strain) at $\varepsilon_{a}=0.8 \%$.

2: The A, B \& $\mathrm{H}$ samples with a pure Ag matrix show an extremely sharp decrease in $I_{C}$ if the $\varepsilon_{2 \%}$ limit is passed. The maximum relative slope is determined as $\approx 500$ at $\varepsilon_{a}=0.47 \%$.

In axial-strain experiments on similar samples, using a classical axial-pull setup, a correlation is found between the mechanical strength of the wires and the type of $I_{C}$ decrease. Wires that are classified here as of the second kind, with a sharp $I_{c}\left(\varepsilon_{a}\right)$ dependence after $\varepsilon_{2 \%}$, also show an irregularity in the stress versus strain behaviour. An almost proportional dependence is found for small strain values indicating an elastic behaviour: $E=d \sigma / d \varepsilon_{a}$. For strains above $\varepsilon_{2 \%}$ the wire starts to yield and the axial stress $(\sigma)$ increases with a smaller slope: $d \sigma / d \varepsilon_{a}$. In wires that are classified in the third group there is not such a distinct yield point and the stress increases more gradually.

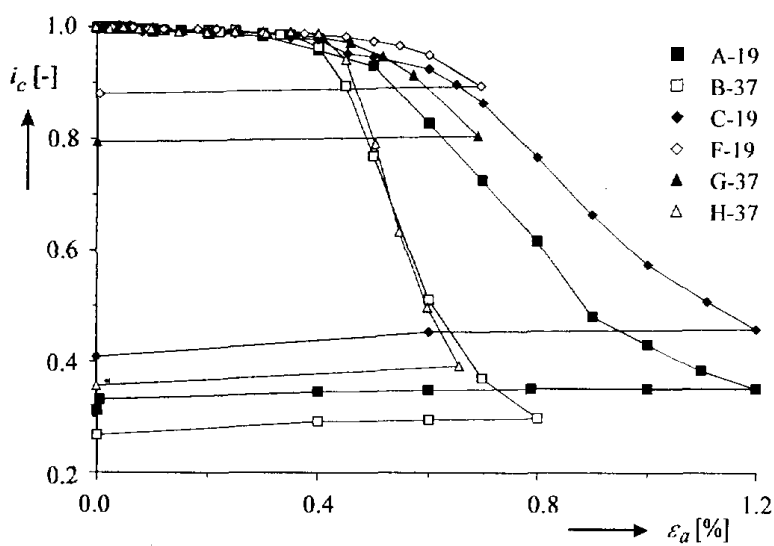

Fig. 1. A comparison of the axial-strain dependence of the normalised critical current in multifilamentary Bi-2212 wires. The samples were investigated at $4.2 \mathrm{~K}$ and a magnetic field of $B=16 \mathrm{~T}$, except $\mathrm{G}-37$ at $B=8 \mathrm{~T}$

\section{B. Low Tensile Strain}

Besides the $I_{c}$ reduction for strains above $\varepsilon_{2 \%}$ it is also interesting to consider the strain regime where the $I_{c}$ reduction is small $(<2 \%)$. If this part of the $I_{c}\left(\varepsilon_{a}\right)$ dependence is magnified then a convincing $I_{c}$ reduction is visible, as can be seen in figure 2 . Note that the reproducibility of the $I_{c}$ is typically $0.3 \%$, due to the voltage noise and the low $n$-value. This implies that for tensile strains larger than $0.05 \%$ a smal: but significant $I_{c}$ reduction is observed.
The reduction of the normalised critical current due to a small axial $\left(\varepsilon_{a}<\varepsilon_{2 \%}\right)$ strain can be described by a constant slope $\left(C_{m}\right)$ :

$i_{c}=1-C_{m} \varepsilon_{a}$,

and a value for $C_{m}=4 \pm 1$. Two possible mechanisms for the small slope of about 4 are an $I_{c}$ reduction of the superconductor due to hydrostatic pressure dependence of the critical temperature $\left(T_{C}\right)$ and the influence of geometrical changes on the $I_{c}$. Both mechanisms are considered here:

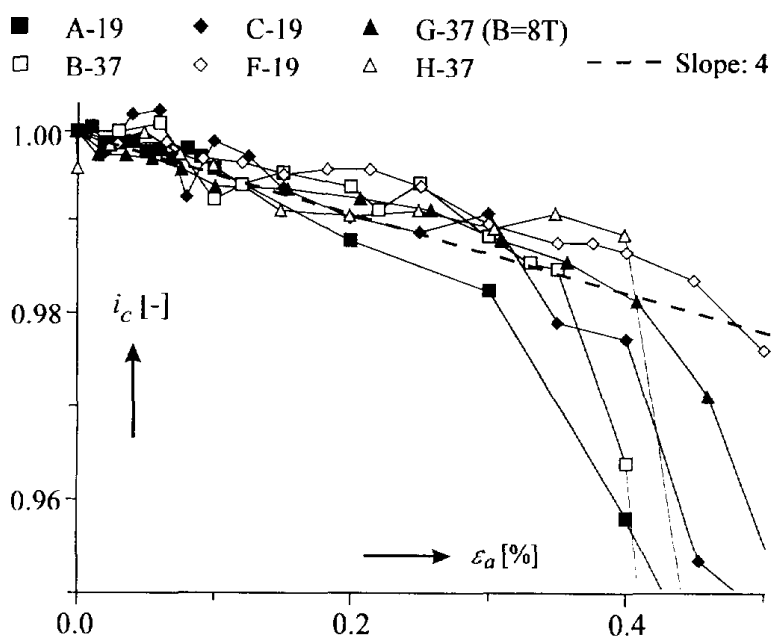

Fig. 2. A magnification of the top part of the normalised $I\left(\varepsilon_{a}\right)$ dependence. The dotted line indicates a reduction with a factor of $4 \times$ the applied strain.

1) Hydrostatic pressure and $T_{c}$ : In the elastic limit a change of $\varepsilon_{a}$ causes a hydrostatic strain of $\varepsilon_{\text {hyd }}=(1-2 u) \varepsilon_{a}$ which again will generate a hydrostatic pressure of:

$$
P=\varepsilon_{a} E .
$$

Where $E$ is the Young's modus. With an $E$ value for $\mathrm{Bi}-2212$ of $20 \mathrm{GPa}$ and a $d T_{c} / d P$ of $0.19 \mathrm{~K} / \mathrm{GPa}$ (surveyed in [4]) the change in the critical temperature is:

$\frac{d T_{c}}{d \varepsilon_{a}}=E \frac{d T_{c}}{d P}=3.8 \mathrm{~K}$

The value calculated here is very small and corresponds to a relative slope of only 0.04 for a $T_{c}$ of $85 \mathrm{~K}$. This relative slope is almost 100 times smaller than the relative slope in $I_{c}$. Moreover, a reversible reduction could be expected if the hydrostatic strain component that effects $T_{c}$ causes the critical-current reduction. It can be concluded that hydrostatic pressure is not relevant for explaining the observed initial slope in $I_{c}\left(\varepsilon_{a}\right)$ 
2) Geometrical changes: A second explanation for the first slope in the current reduction are changes in the geometry of the conductor. In order to evaluate this a onedimensional elastic conductor model is considered with a constant $I_{c}$ along the axis. Two terms will cause a change in $I_{c}$, and a relative change of the length with $\varepsilon_{a}$ will affect the voltage proportionally. If the $U(I)$ dependence is described with a constant $n$-value: $U \propto I^{n}$, then the determined critical current is reduced by $\varepsilon_{\alpha} / n$. The second term is the crosssectional area reduction. The area is reduced by a factor of ( $1-2 v \varepsilon_{a}$ ) which scales proportional with the $I_{c}$. The change in the reduced critical current then has a slope of:

$$
\frac{d i_{c}}{d \varepsilon_{a}}=-\left(\frac{1}{n}+2 v\right)
$$

A measured $n$-value of 4 and an overall value of the Poisson's ratio of 0.25 for the entire wire, gives a slope of -0.75 which is significantly smaller than measured $(=-4)$.

As the descriptions above fail to predict the major part of the $I_{c}$ reduction for small tensile strains, other sources for this reduction have to be considered. The most probable cause are mechanical defects such as certain (micro-)cracks in the polycrystalline $\mathrm{Bi}-2212$ structure. These kind of defects can also explain the absence of any recovery after changing a strain. It is important to determine the mechanism that causes this type of $I_{c}$ reduction more precisely, because it is related to the process that limits the critical current of polycrystalline $\mathrm{Bi}-2212$.

\section{REPETITIVE TENSILE STRAIN}

In the case of practical applications it is recommended to limit the applied axial strains to the "safe" strain range between 0 and $\varepsilon_{2 \%}$. If this strain range is exceeded a strong $I_{c}$ reduction can be expected. The next question that arises is what will happen to the $I_{c}$ if the strain is varied multiple times within a certain strain regime between 0 and $\varepsilon_{2 \%}$. The strain range from 0 to $0.2 \%$ is equivalent to the thermal strain in the $\mathrm{Bi}-2212$ material between the operating and room temperature. Two samples are cycled multiple times in the strain range from 0 to $0.2 \%$. The scaled $I_{c}$ reduction is presented in figure 3.

During the first four cycles the $I_{c}$ reduction in both the samples is similar and a small but continuous reduction is observed. The slope of the $I_{c}$ reduction decreases when the number of cycles is increased. The reduction measured in the B-37 sample indicates a saturation of the $I_{c}$ at a value of about $96 \%$ after 30 cycles. The $I_{c}$ in the $\mathrm{C}-19$ sample shows an abrupt reduction between 4 and 8 cycles of $5 \%$. The size of this step $(5 \%)$ is equivalent to the current that is running through a single filament. For a larger number of cycles the $I_{\mathcal{C}}$ value also seems to saturate at about $85 \%$ of the initial critical current. This experiment with a repetitive load proves that the wire is already slightly damaged when small axial strains of about $0.2 \%$ strain are applied. Another consequence of this observation is that the critical current of this type of Bi-2212 samples will degrade with about the same percentage when they are thermally cycled. A promising feature, for practical applications of these wires, is that the $I_{c}$ reduction seems to saturate at a high number of cycles and at a reasonable high value.

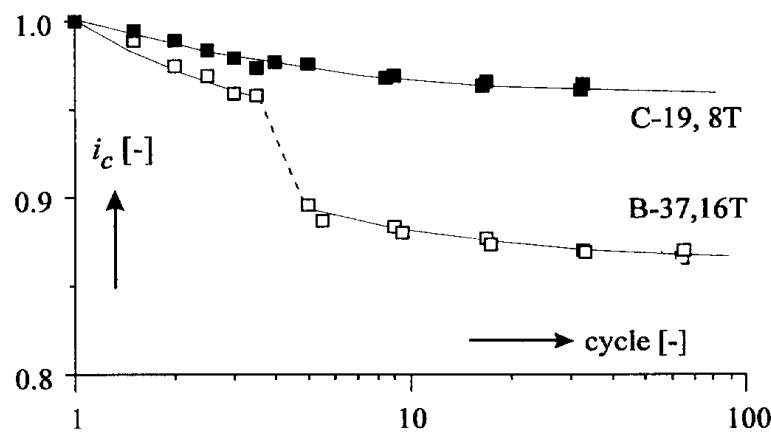

Fig. 3. The scaled $I_{c}$ versus the number of strain cycles for a strain from 0 to $0.2 \%$, presented for two $\mathrm{Bi}-2212$ wires. The initial critical current is defined as cycle number 1 , then $0.2 \%$ strain is cycle number 1.5 and so on.

\section{COMPRESSIVE STRAIN}

The compressive axial-strain dependence of $\dot{I}_{c}$ is investigated in the same $\mathrm{Bi}-2212$ conductor samples. No references are known where such an experiment is described on a $\mathrm{Bi}$ 2212 conductor. In principle an increase of the $I_{c}$ can be expected if the samples are pressed, because of the positive $d T_{c} / d P$ that is measured on the pure Bi-2212 material. According to the calculation in the previous section, a very small slope $d T_{c} / d \varepsilon_{a}=3.8 \mathrm{~K}$ is expected. On the other hand mechanical defects in the $\mathrm{Bi}-2212$ structure may overrule this small increase in $T_{c}$. In figure 4 the $I_{c}\left(\varepsilon_{\alpha}\right)$ is presented for two samples that are subjected to a relatively large axial compression of $2 \%$.

The reduction of the normalised critical current can be described by a constant relative slope in the entire investigated compressive strain regime. This results in an exponential dependence described as:

$\frac{d I_{c}}{I_{c}}=C^{-} \cdot d \varepsilon_{a} \Leftrightarrow i_{c}=\frac{I_{c}}{I_{c}(0)}=e^{C^{-} \cdot \varepsilon_{a}}$

This function is drawn as a solid line in figure 4 with $C^{-}=30$ and a good agreement with the experimental data is observed.

A survey of the compressive axial strain results on the $I_{c}$ is presented in figure 5 . There is a correlation with the behaviour observed in the tensile axial-strain experiments. The A-series shows a strain dependence that deviates from most of the other samples. Around the average slope of $\mathrm{C}^{-}=30$, 
the A-samples show an enhanced degradation, while the $G$ sample show a less pronounced degradation. The observed stronger degradation in the A-series correlates to the stronger decrease that is also observed in axially-strained samples. Within the range of practical interest $\left(-0.4 \%<\varepsilon_{a}<0\right)$ the $I_{c}\left(\varepsilon_{a}\right)$ curve of $\mathrm{Bi}-2212$ under compressive strain can be characterised by a slope of $30 \pm 3$.

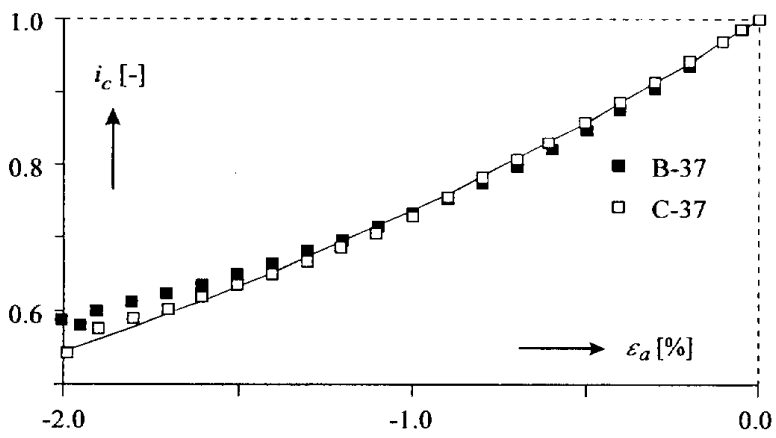

Fig. 4. The $I_{c}\left(\varepsilon_{\alpha}\right)$ measured in two axially compressed $\mathrm{Bi}-2212$ samples: B-37 and C-37 determined at $4.2 \mathrm{~T}$ and $\mathrm{B}=8 \mathrm{~T}$. The solid line represents an exponential reduction of the normalised $I_{c}$ with an exponent of 30 .

The qualitative behaviour of the $I_{c}$ as a function of the axial compression differs from the $I_{C}$ reduction measured in an elongated $\mathrm{Bi}$-wire. There exists not a distinct strain value such as $\varepsilon_{2 \%}$ from where the slope of the $I_{c}$ reduction increases. Obviously there is a principle difference between the current reduction mechanism for both types of axial strain. Even if the zero point of the strain is shifted to the position where the axial strain in the Bi-2212 filaments is cancelled (at $\varepsilon_{a} \approx 0.4 \%$ ), as is common practice in $\mathrm{Nb}_{3} \mathrm{Sn}$ conductors, the $I_{c}\left(\varepsilon_{z}\right)$ curve remains different for positive and negative strains inside the filaments.
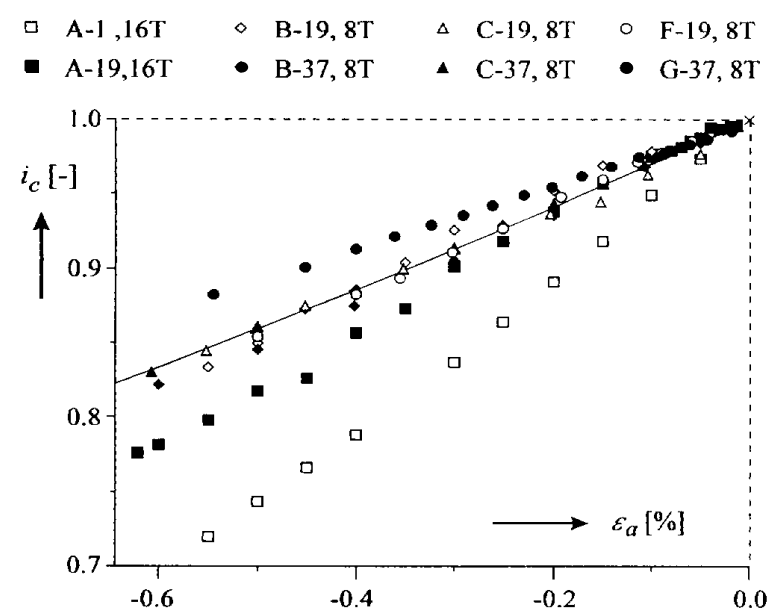

Fig. 5. The normalised critical current versus compressive axial strains, measured at $4.2 \mathrm{~K}$ and $\mathrm{B}=8$ and $16 \mathrm{~T}$

\section{CONCLUSIONS}

1. The critical-current dependence of $\mathrm{Bi}-2212$ wires with a Ag matrix, subjected to both compressive and tensile axial strains, is measured on a U-shaped bending spring. Only negative changes of the critical current are found after changing the strain in any direction (= irreversible).

2. A small slope, of approximately 4 in the relative current reduction, appears in the first part of the tensile axial strain range. This $I_{c}$ reduction cannot be explained by normal changes in the geometry of the conductor nor by the influence of hydrostatic strain on the critical temperature.

3. The measured behaviour of the $I_{c}$ reduction indicates that the $I_{c}$ reduction is determined by mechanical damage in the granular Bi-2212 material. The most probable cause is the appearance of cracks in the polycrystalline structure.

4 . The relative $I_{c}$ reduction decreases to zero if a small repetitive strain is applied. In the strain range, equivalent to a typical thermal cycle from 300 to $4.2 \mathrm{~K}$, the $I_{c}$ reduction may saturate at a value of about $90 \%$ of the initial value.

5 . A regular and irreversible $I_{c}$ reduction is observed when the wire is compressed in the axial direction. In these samples the reduction is described by a slope of $30 \pm 3$.

6. As illustrated in figure 6 the entire investigated axial strain range can be subdivided in three parts: I - compressive, II - small tensile $\left(0<\varepsilon_{a}<\varepsilon_{2 \%}\right)$ and III - large tensile strain $\left(\varepsilon_{a}>\varepsilon_{2 \%}\right)$. A different, but always irreversible, strain reduction is measured in these three strain regimes.

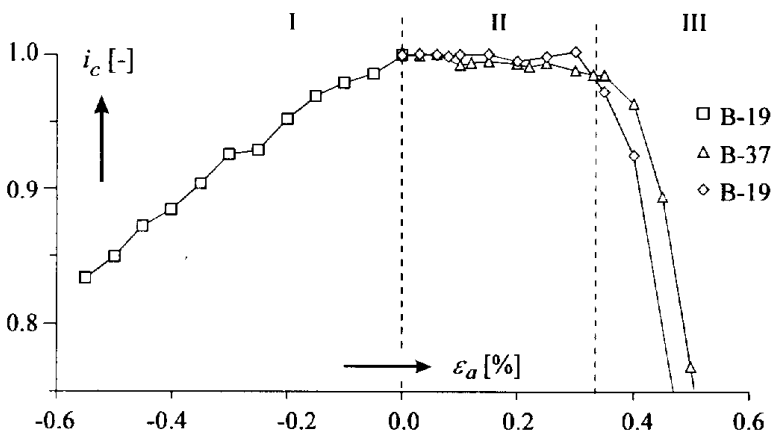

Fig. 6. The normalised $I_{c}$ for compressive and tensile axial strains at $4.2 \mathrm{~K}$.

\section{REFERENCES}

[1] J. Tenbrink et. Al., "Development of high-T ${ }_{c}$ superconducting wires for magnet applications", IEEE Trans. Mag. vol. 27, 1991, pp. 1239.

[2] B. ten Haken, R.B. Wilson, H.H.J. ten Kate and J. Tenbrink, "Critical current reduction due to strain in Bi-2212 wires", in: "Applied superconductivity", editor: H.C. Freyhardt, DGM Informationsgesellschaft mbH, Germany, 1993, p. B235

[3] J.W. Ekin et. al., "Improved uniaxial strain tolerance of the critical current measured in $\mathrm{Ag}$-sheated $\mathrm{Bi}_{2} \mathrm{Sr}_{2} \mathrm{Ca}_{1} \mathrm{Cu}_{2} \mathrm{O}_{8+x}$ superconductors", Adv. in Cryo. Eng. vol. 38, 1992, pp. 1041 .

[4] B. ten Haken, "Strain effects on the critical properties of high-field superconductors", Phd. Thesis, University of Twente, Enschede, The Netherlands, 1994 